\title{
FROBENIUS NORM INEQUALITIES OF COMMUTATORS BASED ON DIFFERENT PRODUCTS
}

\author{
Wei-Hui Liu, Ze-Jia Xie And XiaO-Qing Jin*
}

Abstract. The difference $A B-B A$ of two matrices $A$ and $B$ is called the commutator (or Lie product). In this paper, we are concerned with inequalities for the Frobenius norm of commutators based on other products, including the Kronecker product, the Khatri-Rao product, the contracted product, and the T-product. We also study the characterization of their corresponding maximal pairs.

Mathematics subject classification (2020): 15A52, 15A45, 60H25, 65F35.

Keywords and phrases: Commutator, Böttcher-Wenzel conjecture, Frobenius norm, Kronecker product, Khatri-Rao product, contracted product, T-product, maximal pair.

\section{REFERENCES}

[1] P. Absil, R. Mahony, R. Sepulchre, Optimization Algorithms on Matrix Manifolds, Princeton University Press, Princeton, 2008.

[2] K. AudenAERT, Variance bounds, with an application to norm bounds for commutators, Linear A1gebra Appl., vol. 432 (2010), pp. 1126-1143.

[3] B. BADER, T. KOLDA, Algorithm 862: MATLAB tensor classes for fast algorithm prototyping, ACM Trans. Math. Software, vol. 32 (2006), pp. 635-653.

[4] R. Bhatia, Matrix Analysis, Springer, New York, 1997.

[5] R. Bhatia, F. Kittaneh, Commutators, pinchings and spectral variation, Oper. Matrices, vol. 2 (2008), pp. 143-151.

[6] A. BötTCHER, D. WEnZEL, How big can the commutator of two matrices be and how big is it typically?, Linear Algebra Appl., vol. 403 (2005), pp. 216-228.

[7] A. Böttcher, D. Wenzel, Frobenius norm and the commutator, Linear Algebra Appl., vol. 429 (2008), pp. 1864-1885.

[8] C. Cheng, K. Fong, W. LEI, On some norm inequalities involving the commutator and $X Y-Y X^{T}$, Linear Algebra Appl., vol. 438 (2013), pp. 2793-2807.

[9] C. Cheng, K. Fong, I. LOK, Another proof for commutators with maximal Frobenius norm, Recent Advances in Scientific Computing and Matrix Analysis, pp. 9-14, Editors: X. Jin, H. Sun, and S. Vong, International Press, 2011.

[10] C. Cheng, R. Jiao, Proof of Wenzel's conjecture concerning singular values of the commutator of rank one matrices, Linear Algebra Appl., vol. 592 (2020), pp. 165-174.

[11] C. CHENG, X. Jin, S. Vong, A survey on the Böttcher-Wenzel conjecture and related problems, Oper. Matrices, vol. 9 (2015), pp. 659-673.

[12] C. Cheng, C. LEI, On Schatten p-norms of commutators, Linear Algebra Appl., vol. 484 (2015), pp. 409-434.

[13] C. CHENG, Y. LIANG, Some sharp bounds for the commutator of real matrices, Linear Algebra Appl., vol. 521 (2017), pp. 263-282.

[14] C. Cheng, Y. LiAng, Singular values, eigenvalues and diagonal elements of the commutator of $2 \times 2$ rank one matrices, Electron. J. Linear Algebra, vol. 36 (2020), pp. 1-20.

[15] C. Cheng, S. Vong, D. Wenzel, Commutators with maximal Frobenius norm, Linear Algebra Appl., vol. 432 (2010), pp. 292-306. 
[16] A. Cichocki, N. Lee, I. Oseledets, A. Phan, Q. ZhaO, D. Mandic, Low-rank tensor networks for dimensionality reduction and large-scale optimization problems: perspectives and challenges part 1, ArXiv preprint, arXiv:1609.00893v1, 2016.

[17] L. EldÉN, B. SAVAS, A Newton-Grassmann method for computing the best multilinear rank$\left(r_{1}, r_{2}, r_{3}\right)$ approximation of a tensor, SIAM J. Matrix Anal. Appl., vol. 31 (2009), pp. 248-271.

[18] K. Fong, C. CHeng, I. LoK, Another unitarily invariant norm attaining the minimum norm bound for commutators, Linear Algebra Appl., vol. 433 (2010), pp. 1793-1797.

[19] K. FONG, I. LOK, C. CHENG, A note on the norm of commutator and the norm of $X Y-Y X^{T}$, Linear Algebra Appl., vol. 435 (2011), pp. 1191-1201.

[20] N. HaO, M. Kilmer, K. Braman, R. Hoover, Facial recognition using tensor-tensor decompositions, SIAM J. Imaging Sci., vol. 6 (2013), pp. 437-463.

[21] X. Jin, S. Vong, An Introduction to Applied Matrix Analysis, Higher Education Press, Beijing, 2016.

[22] C. KHATRI, C. RAO, Solutions to some functional equations and their applications to characterization of probability distributions, Sankhya, vol. 30 (1968), pp. 167-180.

[23] M. Kilmer, K. BRAmAn, N. HAO, R. Hoover, Third-order tensors as operators on matrices: a theoretical and computational framework with applications in imaging, SIAM J. Matrix Anal. Appl., vol. 34 (2013), pp. 148-172.

[24] M. Kilmer, C. Martin, Factorization strategies for third-order tensors, Linear Algebra Appl., vol. 435 (2011), pp. 641-658.

[25] F. Kittaneh, Commutator inequalities associated with the polar decomposition, Proc. Amer. Math. Soc., vol. 130 (2002), pp. 1279-1283.

[26] F. KitTANEH, Inequalities for commutators of positive operators, J. Funct. Anal., vol. 250 (2007), pp. $132-143$.

[27] L. LÁsZLó, Proof of Böttcher and Wenzel's conjecture on commutator norms for 3-by-3 matrices, Linear Algebra Appl., vol. 422 (2007), pp. 659-663.

[28] I. LoK, Norm inequalities for a matrix product analogous to the commutator, M. Sc. Thesis, University of Macau, 2010.

[29] Z. Lu, Proof of the normal scalar curvature conjecture, ArXiv preprint, arXiv:0711.3510v1 [math.DG], 2007.

[30] Z. LU, Normal scalar curvature conjecture and its applications, J. Funct. Anal., vol. 261 (2011), pp. 1284-1308.

[31] Z. Lu, Remarks on the Böttcher-Wenzel inequality, Linear Algebra Appl., vol. 436 (2012), pp. 25312535.

[32] Z. Lu, D. Wenzel, Commutator estimates comprising the Frobenius norm - looking back and forth, in: D. Bini, T. Ehrhardt, A. Karlovich, I. Spitkovsky (Eds.), Large Truncated Toeplitz Matrices, Toeplitz Operators, and Related Topics, in: Operator Theory: Advances and Applications, vol. 259, Birkhäuser, Cham, 2017, pp. 533-559.

[33] K. LunD, The tensor t-function: a definition for functions of third-order tensors, ArXiv preprint, arXiv: $1806.07261,2018$.

[34] S. Ragnarsson, C. VAn Loan, Block tensors unfoldings, SIAM J. Matrix Anal. Appl., vol. 33 (2012), pp. 149-169.

[35] C. RAO, Estimation of heteroscedastic variances in linear models, Journal of the American Statistical Association, vol. 65 (1970), pp. 161-172.

[36] S. Vong, X. Jin, Proof of Böttcher and Wenzel's conjecture, Oper. Matrices, vol. 2 (2008), pp. 435442.

[37] Z. XIE, C. CHENG, X. Jin, Some norm inequalities for commutators of contracted tensor products, Linear Algebra Appl., vol. 540 (2018), pp. 244-256.

[38] X. Zhan, Matrix Theory, American Mathematical Society, Rhode Island, 2013. 